\title{
Fast continuous model of Shack-Hartmann wavefront sensors for atmospheric tomography on ELTs
}

\author{
Fabien Momey and Michel Tallon
}

Université de Lyon, Lyon, F-69003, France ; Université Lyon 1, Observatoire de Lyon, 9 avenue Charles André, Saint-Genis Laval, F-69230, France ; CNRS, UMR 5574, Centre de Recherche Astrophysique de Lyon ; Ecole Normale Supérieure de Lyon, Lyon, F-69007, France

\begin{abstract}
Atmospheric tomography is a key element for many adaptive optics architectures on which the coming Extremely Large Telescopes rely. When modeling atmospheric tomography, the samples of the wavefronts in the turbulent layers are not aligned with the subapertures after propagation down to the wavefront sensors (WFS). So the classical models of Shack-Hartmann WFS (e.g. Fried's model) include resampling before performing the standard slopes calculation. This operation introduces errors and only approximates the response of the WFS (error of 10 to $20 \%$ of the standard deviation of the slopes). We introduce a continuous model that represents the wavefronts on a basis of continuous functions in the turbulent layers. Propagation and gradient measurements are then computed analytically, without any resampling. Assuming the separability of the basis functions on the two dimensions, we obtain a sparse operator that can be factorized in two components. This factorization enhances the speed close to that of the standard approach using interpolation. We obtain a fast accurate continuous WFS model suitable to wide field adaptive optics systems on ELTs.
\end{abstract}

\section{Introduction}

Besides a very significant increase in size, the coming Extremely Large Telescopes (ELTs) rely on adaptive optics (AO) to achieve outstanding performances. Various AO architectures are currently studied to optimize the ELTs for different science cases [1]. The need of an efficient atmospheric tomography [2,3] is critical for several of these architectures, like laser tomography AO, multiconjugate $\mathrm{AO}$, or multi-object $\mathrm{AO}[1]$.

When modeling a classical single conjugate AO, the sampling grid of the single layer is chosen aligned with the subapertures of a Shack-Hartmann wavefront sensor (WFS); for instance each sample is located at a subaperture corner for Fried's geometry [4]. For atmospheric tomography, modeling wavefronts propagation down to the wavefront sensors yields misalignments between the wavefront samples and the corners of the subapertures, depending on the position of the guide star in the field of view and the altitude of the layer. Furthermore, the sampling step of the propagated grid is not the same when considering laser guide stars or natural guide stars. Therefore resampling is necessary before computing wavefront measurements with a classical approach. This resampling step entails additional modeling errors in the reconstruction process. To avoid any resampling error, we introduce a so-called "continuous model". We model the wavefronts on a basis of continuous functions, and we compute analytically the propagation of the layers and the WFS measurements.

But such a continuous model cannot be implemented for an ELT without considering computational load. The large size of the coming ELTs yields a dramatic increase of the number of linear equations to be solved for running an adaptive optics (AO) system in real time, particularly where atmospheric tomography is involved. For instance, considering orders of magnitude, modeling 10 layers and 10 wavefront sensors with $100 \times 100$ subapertures would lead to $2 \times 10^{5}$ equations and $10^{5}$ unknowns typically. So the implementation of the continuous model must not increase significantly the number of operations and must use little memory space. This is why we use separable basis functions for the wavefront modeling. We then obtain a sparse operator that can be factorized in two components, acting respectively on each dimension of the wavefront. This factorization allows this modeling to be quickly performed.

\footnotetext{
a e-mail: fabien.momey@obs.univ-lyon1.fr
} the original work is properly cited. 


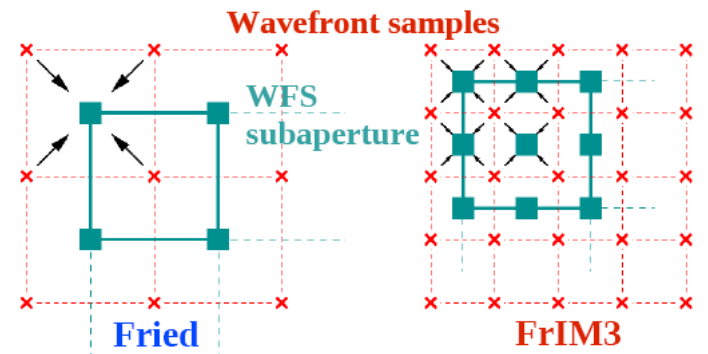

Fig. 1. Layout of the two considered models with interpolation. The turbulence layer is sampled on the crosses. The WFS models need the interpolated values on the square points.

In Section 2, a quantitative study of the interpolation errors on slopes measurements is presented. Section 3 introduces the formalism of the continuous model. In Section 4, the number of operations and the reconstruction errors of the continuous model are compared with two other models using interpolation.

\section{Evaluation of interpolation errors on WFS measurements}

The aim is here to estimate the modeling errors on the WFS measurements when layer samples are interpolated for using a WFS model based on finite differences. We consider two models of this kind (Fig.1):

- Fried's model [4].

- The so-called FrIM3 model based on sampling the subapertures 2 times finer than Fried's model (Fig.1).

We define the sampling rate of the layer as the number of samples per subaperture size, in one direction. Thus a sampling rate of 1 means that the sampling step in $x$ and $y$ directions is equal to the size of a subaperture. A Kolmogorov wavefront is drawn on this grid, randomly shifted. The obtained values on this sampling grid are considered as the coefficients of a linear combination of bilinear basis functions that define a continuous wavefront over the pupil. The WFS measurements are then computed analytically from this continuous wavefront.

In the case of a perfect alignment of the wavefront samples with the corners of the subapertures, this continuous model leads to the usual Fried's model. But the wavefront being given on the set of basis functions, the continuous model computes the perfect measurements for any sampling rate.

For each sampling rate, the noise-free WFS measurements are computed for a $64 \times 64$ WFS for the continuous model and for the 2 tested models, Fried and FrIM3. Taking the continuous model measurements as the reference, the root mean square error on the measurements is computed for the 2 interpolated models, normalized by the standard deviation of the continuous model slopes.

Figure 2 shows that the relative rms error increases with the sampling rate. For low sampling rates (typically Fried's sampling), the errors are dominated by interpolation errors. These errors vanish as the sampling rate increases, where WFS modeling errors dominate and converge to an asymptotic value. Since the WFS models are different, aliasing errors are not exactly the same and contribute to modeling errors. The relative rms error of Fried's model is in the range $10 \%-20 \%$ in the typical sampling rates. With FrIM3 model the relative rms error is $\sim 4 \%$ only in the same range. This error is to be added to the measurement noise.

\section{Continuous model}

As explained before, atmospheric tomography needs a modeling of the propagation of the corrugated wavefront from a sampled atmospheric layer down to the wavefront sensor. This implies misalignements of the samples with the edges of the subapertures depending on the position and type (natural 


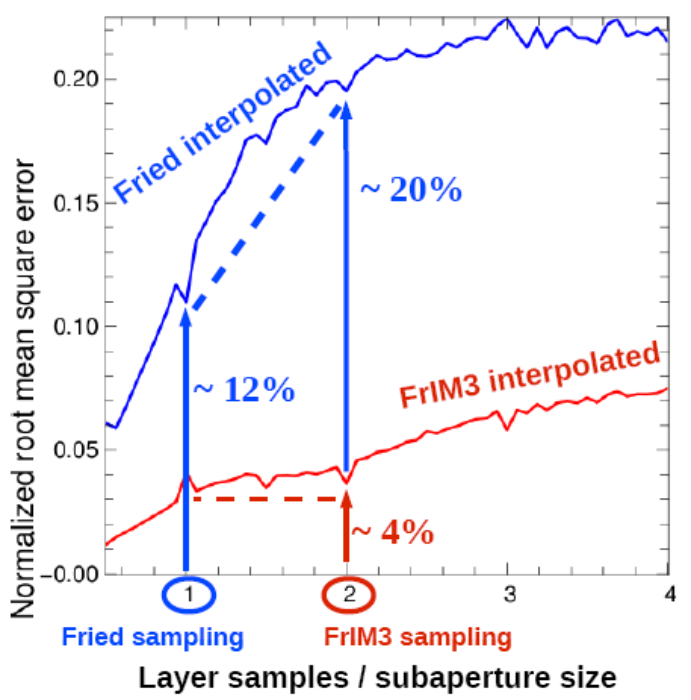

Fig. 2. Root mean square modeling error on the WFS measurements (slopes) for the two models with interpolation, normalized by the standard deviation of the continuous model slopes (reference). A single layer is randomly shifted, with increasing sampling rate. Slopes measurements are computed without noise.

or laser) of the guide star in the field of view and the altitude of the layer. As a result, a step of interpolation is implemented before applying usual WFS models like Fried's model. This resampling process adds a noise to the WFS measurements, as shown in Section 2.

The continuous model is introduced to avoid these modeling errors. The wavefronts are represented as a linear combination of basis functions. An atmospheric layer $L$ is represented by:

$$
\begin{aligned}
\varphi_{L}(x, y)=\sum_{k=1}^{n_{k}} \sum_{l=1}^{n_{l}} w_{k l} b_{k l}(x, y) & =\sum_{k=1}^{n_{k}} \sum_{l=1}^{n_{l}} w_{k l} b_{k}(x) b_{l}(y) \\
& =\sum_{k=1}^{n_{k}} \sum_{l=1}^{n_{l}} w_{k l} b\left(\frac{x-c_{x k}}{r_{x}}\right) b\left(\frac{y-c_{y l}}{r_{y}}\right) .
\end{aligned}
$$

$\varphi_{L}(x, y)$ and $w_{k l}$ are optical path differences (measured in meters) added to the incident wavefront. $r_{x}$ and $r_{y}$ are the radius of the support of the functions along the $x$ - and $y$-axis respectively. $w_{k l}$ is the amplitude of each function, as illustrated by Figure 3. For bilinear functions, $w_{k l}$ is also the value of the corresponding sample of the wavefront.

Since the computational load and the memory space are critical for ELTs, we have limited the possibilities to separable basis functions in Eq. (1), as it is usually the case for modeling deformable mirrors [5].

The aim is then to compute analytically the propagation of the layers and the gradients from this modeling. For a subaperture whose opposite corners are at coordinates $\left(x_{i}, y_{i}\right)$ and $\left(x_{i+1}, y_{i+1}\right)$ (Fig.3), the $x$-slopes are calculated as follows:

$$
\begin{aligned}
M_{x}\left(x_{i}, y_{j}\right) & =\frac{1}{\mathcal{A}_{i j}} \iint_{A_{i j}} \mathrm{~d} x \mathrm{~d} y \frac{\partial}{\partial x} \varphi_{L}(x, y) \\
& =\sum_{k=1}^{n_{k}} \frac{1}{\left|x_{i+1}-x_{i}\right|} \int_{x_{i}}^{x_{i+1}} \mathrm{~d} x \frac{\partial}{\partial x} b_{k}(x) \sum_{l=1}^{n_{l}} w_{k l} \frac{1}{\left|y_{j+1}-y_{j}\right|} \int_{y_{i}}^{y_{j+1}} \mathrm{~d} y b_{l}(y) \\
& =\sum_{k=1}^{n_{k}} Z_{x i k}^{-} \sum_{l=1}^{n_{l}} w_{k l} \nabla_{y_{j l}}^{+}
\end{aligned}
$$




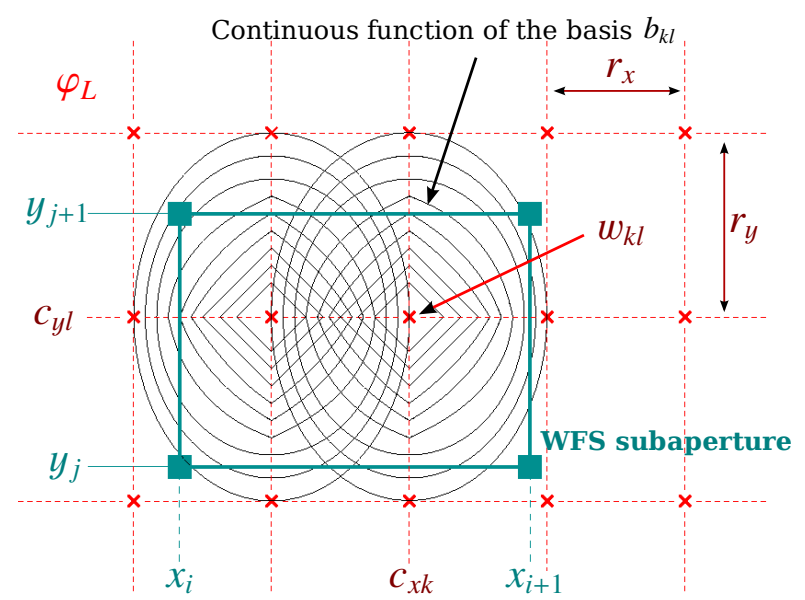

Fig. 3. Layout of the continuous model.

where $\mathcal{A}_{i j}$ is the area of one subaperture. A similar calculation is performed for the $y$-slopes. The separability of the functions of the basis on the 2 axis allows the integrals to be split. As a result these integrals correspond to the coefficients of two factorized matrices per gradient. We can notice that these matrices are sparse if we choose basis functions with a finite support. Considering the samples $w_{k l}$ as the coefficients of a map $\mathbf{W}$, the matricial form of our continuous operator is:

$$
\mathbf{M}_{x}=\mathbf{Z}_{\mathbf{x}}^{-} \cdot \mathbf{W} \cdot \nabla_{\mathbf{y}}^{+\mathrm{T}} \text { and } \quad \mathbf{M}_{y}=\nabla_{\mathbf{x}}^{+} \cdot \mathbf{W} \cdot \mathbf{Z}_{\mathbf{y}}^{-\mathrm{T}}
$$

The factorization enhances the speed of the operator, performing less operations. We observed, studying the matrices and their number of non-zero elements, a decrease of $30 \%$ of the number of multiplications per subaperture whatever the sampling of the layer is.

\section{Results}

\subsection{Number of operations}

We need to estimate the computational load when using the continuous model, since on ELTs, we cannot accept a significant increase of the number of operations compared to the models with interpolation. For a comparison, we compute the number of MACs (Multiply-ACcumulate) per subaperture, which are the common operations that compute the product of two numbers and add the result to an accumulator.

The number of MACs is computed as a function of the number of layers for 2 particular samplings: Fried (sampling step = subaperture size) and FrIM3 (sampling step = half subaperture size) (Fig 1). Two steps can be discriminated:

- Interpolation. Fried's geometry requires 4 MACs per subaperture per layer and FrIM3's geometry requires 16 MACs per subaperture per layer. This models the propagation of each layer down to the same sampling grid in the pupil plane.

- Slopes computation. This step is done only once after all the propagated layers are added altogether. Fried's geometry requires 8 MACs per subaperture and FrIM3's geometry requires 12 MACs per subaperture.

Figure 4 compares the number of MACs per subaperture required by the continuous model to the usual interpolated models, Fried and FrIM3. From 2 to 10 layers in Fried sampling, the continuous model requires $50 \%$ to $140 \%$ more MACs per subaperture than the interpolated Fried's model. From 2 to 10 layers in FrIM3 sampling, it requires $10 \%$ to $40 \%$ more MACs per subaperture than the interpolated FrIM3's model. Therefore we stay in an acceptable amount of required operations for atmospheric tomography. 


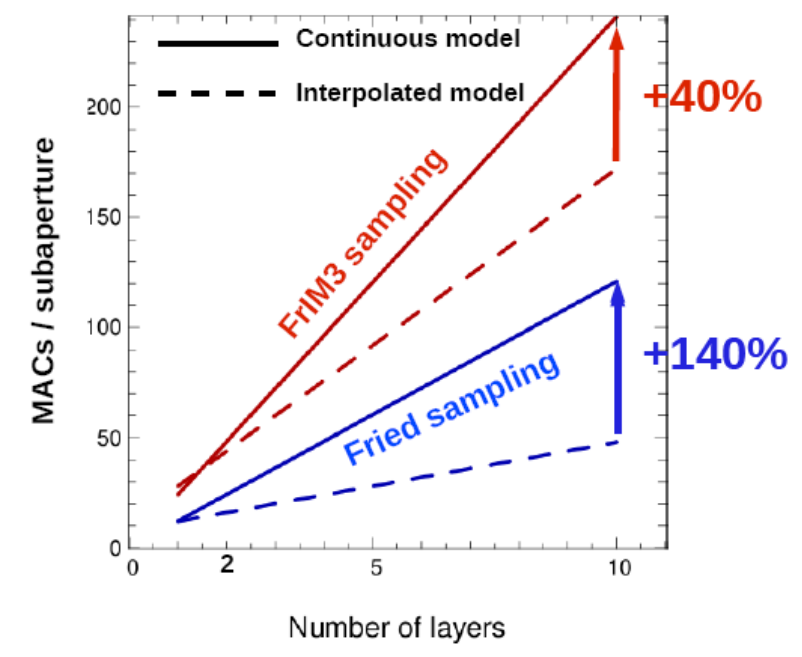

Fig. 4. Number of MACs per subaperture required by the continuous model as a function of the number of layers, compared to the interpolated models, Fried's model (blue dashed curve) and FrIM3 (red dashed curve).

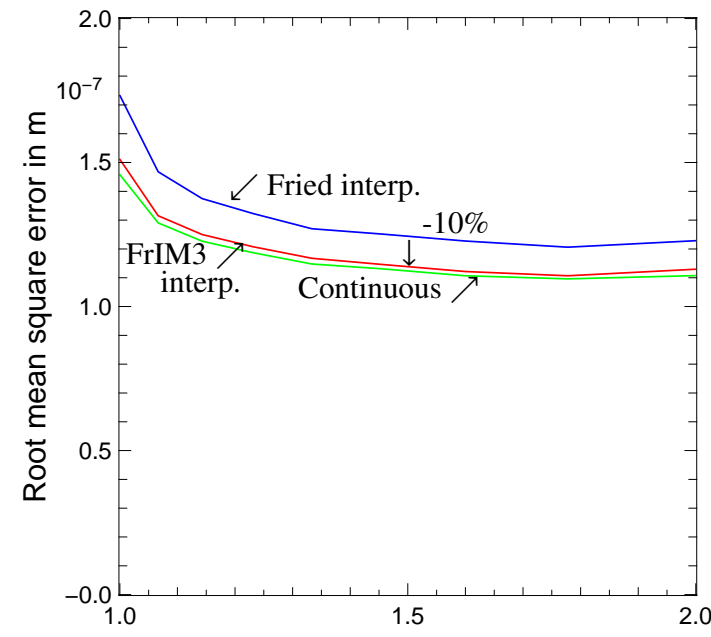

Layer samples per subaperture size

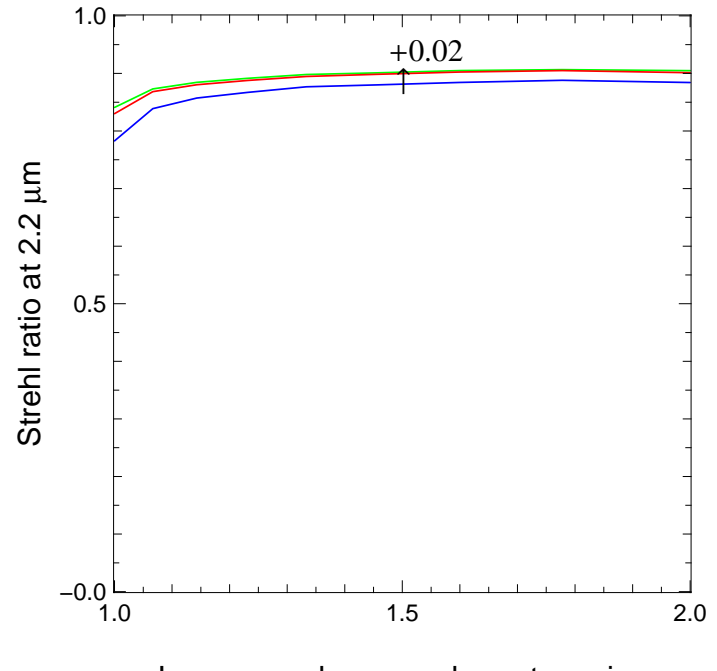

Layer samples per subaperture size

Fig. 5. Evaluation of the total reconstruction errors for the three WFS models: Fried's model, FrIM3, and continuous, the two first ones using interpolation. The reference wavefronts were drawn on a fine grid (16 samples per subaperture size in one direction) and the slopes measurements were computed for a $64 \times 64$ wavefront sensor with a SNR 200 (50nm of rms noise). On the right, the rms errors are converted into Strehl ratio in K band.

\subsection{Comparison of the reconstruction errors}

Finally we compare the reconstruction errors between the 3 models (continuous, Fried, and FrIM3). Since the total error combines the measurement noise with other errors like fitting and aliasing errors, the result depends on their relative contributions and quantitatively changes with the signal-to-noise ratio (SNR) for instance. We present here one case, as an illustration of what can be the benefit of the continous model in the global problem of wavefront reconstruction.

For this simulation, a reference wavefront is drawn on a fine grid with 16 samples per subaperture size, with an optical path difference standard deviation of about $10 \mu \mathrm{m} \mathrm{rms}$, corresponding to 
$D / r_{0}=247$ for Kolmogorov turbulence $\left(D=42 \mathrm{~m}\right.$, and $\left.r_{0}=17 \mathrm{~cm}\right)$. From this reference wavefront, the continuous model is used to simulate the slopes measurements for a $64 \times 64$ wavefront sensor. A noise of $50 \mathrm{~nm} \mathrm{rms}$ is added to these measurements, leading to SNR $\sim 200$. For the modeling, a single layer randomly shifted is defined with sampling rates from Fried sampling to FrIM3 sampling with each of the three WFS models. The reconstruction is performed on this modeling layer.

Figure 5 compares the root mean square errors on the pupil of the three models between the reconstructed and the initial wavefronts. We observe a gain of about $10 \%$ for the continuous model compared to the Fried's model. However we have a marginal gain compared to the FrIM3's model that appears here as a good trade-off between quality and number of operations. The corresponding increase of the $\mathrm{K}$ band Strehl ratio is about $2 \%$ and reaches $90 \%$ (Fig. 5).

\section{Conclusion}

The aim of this paper was to present a study made on the performances of a fast continuous model of Shack-Hartmann wavefront sensor for atmospheric tomography on ELTs. Starting from a modeling of the wavefronts with a basis of continuous functions, it computes analytically the propagation of the layers and the gradients on the subapertures. This avoids modeling errors due to the resampling process involved by misalignments of the samples with the edges of subapertures after propagation of the layers down to the wavefront sensor, in atmospheric tomography. The use of a basis of separable functions over the two axis allows a factorization of the operator, enhancing the speed to performances suitable with ELTs needs in terms of computational load. As a result, on a example, we obtain a gain of about $10 \%$ in the reconstruction errors compared to a standard model (Fried) with interpolation, corresponding to an increase of the K band Strehl ratio from $\sim 88 \%$ to $\sim 90 \%$.

In this study, the continous model proved itself as a useful tool to study the contributions of the various errors in wavefront reconstruction. Future work will include the use of other compact and separable basis functions.

\section{References}

1. N. Hubin, B.L. Ellerbroek, R. Arsenault, R.M. Clare, R. Dekany, L. Gilles, M. Kasper, G. Herriot, M. Le Louarn, E. Marchetti et al., Adaptive optics for Extremely Large Telescopes, in Scientific Requirements for Extremely Large Telescopes, edited by P.A. Whitelock, M. Dennefeld, B. Leibundgut (Cambridge University Press, 2005), Vol. 232 of IAU Symposium, pp. 60-85

2. S. Esposito, C. R. Physique 6(10), 1039 (2005)

3. M. Tallon, R. Foy, Astron. Astrophys. 235(1-2), 549 (1990)

4. D.L. Fried, J. Opt. Soc. Am. 67(3), 370 (1977)

5. M. Le Louarn, C. Vérinaud, V. Korkiakoski, C. R. Physique 6(10), 1070 (2005) 